\title{
Mental Health Issues in Coronavirus Disease 2019 Pandemic: Evolving a Strategy
}

\author{
Vaibhav Patil ${ }^{1}$ Ankit Gupta ${ }^{1}$ Rakesh Kumar Chadda ${ }^{1}$ \\ ${ }^{1}$ Department of Psychiatry, All India Institute of Medical Sciences, \\ Address for correspondence Rakesh Kumar Chadda, MBBS, \\ Ansari Nagar, New Delhi, India \\ MD, FAMS, FRCPsych, DFAPA, Department of Psychiatry, All India \\ Institute of Medical Sciences, Ansari Nagar, New Delhi 110029, India \\ (e-mail: drrakeshchadda@gmail.com).
}

\begin{abstract}
Coronavirus disease 2019 (COVID-19) pandemic has become a global health emergency in a short span of time. Novel kind of the disease, highly infectious nature, rapid progress with serious medical complications and risk of death, and absence of effective treatment and vaccine have all added to a community fear. Need for social distancing, social isolation, and quarantine in contacts further contributes to the fear and also creates a stigma. Declaration of lockdown in many parts of the world to prevent spread of illness has been associated with socioeconomic consequences with great loss to the world economy as well unemployment in a large section of the population. All these factors impose a high risk of mental health problems like anxiety, fear, worries, sleep disturbances, depression, etc. in the general population, and also worsening of the existing symptoms in persons with existing mental illness. The impact of the pan-

Keywords

- COVID-19

- mental health

- management

- lockdown demic on mental health is likely to be long lasting, and a great challenge to the mental health professionals and the policy makers. This paper discusses various strategies to effectively manage the mental health issues in the light of limited availability of mental health resources and restricted access to health services due to lockdown in low-resource settings.
\end{abstract}

\section{Introduction}

On March 11, 2020, the World Health Organization (WHO) declared the novel coronavirus disease 2019 (COVID-19) outbreak a global pandemic. It began as a cluster of cases of pneumonia in Wuhan, Hubei Province, China and started spreading alarmingly all over the world. By May 14, 2020, over 4,218,212 confirmed cases with 290,242 deaths across 216 countries had been reported. ${ }^{1}$ The numbers in India stood at 78,003 cases with 2,549 deaths till the same date. ${ }^{2}$ This rapidly spreading public health emergency has affected health, safety, and well-being of all at individual as well as community level. Novel nature of the virus, its rapid spread with high infectivity, lack of effective treatment, and unpredictable prognosis with restricted health infrastructure have led the local governments to impose nationwide lockdowns by many countries including India as a measure to prevent the chain of transmission. The lockdown resulted into restriction of movements, limited availability of health services, shutting down of educational institutions and public transport along with ban on social gatherings. Agricultural and industrial sectors have been severely affected by the COVID-19 pandemic. This socioeconomic disruption has come with significant impact on the livelihoods of large strata of population, as there is high risk of unemployment and financial loss. Fear of illness, social isolation, and fear and misinformation spreading through social media along with uncertainty about the future have potential of adversely affecting the mental health of the population. Various studies have already demonstrated impact of infectious disease outbreak on mental health in the recent years. ${ }^{3,4}$ The persons affected by the economic shutdown, persons with COVID-19 and their contacts, especially those being kept in quarantine and healthcare workers (HCWs) are at high risk of developing mental health issues. Though at present the primary focus is on physical health, neglecting mental health may have 
deleterious effects in form of increase in anxiety and depressive symptoms, increased use of psychoactive substances, sleep disturbances, and so on. In fact, there have also been a few reports of suicidal attempts and completed suicides in persons being under quarantine or admitted with COVID-19 infection. Thus, the mental health professionals (MHPs) face several challenges in this background, and there is a need to evolve well-planned strategies to meet this challenge.

This paper discusses various strategies at individual as well as the community level to meet the mental health challenges being faced in background of the COVID-19 pandemic.

\section{Challenges for Mental Health during Coronavirus Disease 2019 Pandemic}

COVID-19 has been spreading alarmingly across the globe with fatalities increasing day by day. Restrictions imposed through lockdown leading to financial instability have created a sense of panic in the general population. Social isolation, fear of contracting illness, unpredictability of disease with high morbidity and mortality, fear of unemployment, and sense of loss of control are among many factors contributing to the rise of mental health problems. Though it is early to study impact of COVID-19 systematically, mental health issues like anxiety, worries about self and family getting the infection, depression, sleep disturbance, symptoms of posttraumatic stress disorder, multiple pains, and feeling stressed have been reported in recent studies. ${ }^{5,6}$ People with preexisting mental illness are more likely to react to such stressful situations, leading to relapse or worsening of preexisting mental health condition. Lockdown, lack of public transport services, and limited access to health services have made difficult for people with mental disorders in maintaining their regular follow-up with the mental health services. Persons with chronic or severe physical illnesses are also facing similar issues leading to worsening of their illness and worries. Individuals infected by COVID-19 who are under isolation or treatment or those who have been quarantined may develop severe anxiety, worries, depression and stress reactions. Quarantine and isolation have also been reported to be associated with stigma and discrimination. ${ }^{7}$

There are also vulnerable groups like the elderly, pregnant ladies, children, migrant workers, unemployed people, and individuals with existing severe medical or psychiatric illnesses, who would need extra care. The HCWs are also at high risk of contracting disease particularly those who are involved in diagnosing or treating known or suspected COVID-19 patients. Fear of contracting illness and infecting loved ones along with threat to life, increased work hours, lack of adequate protective equipment, fear of discrimination or violence in society, and lack of effective treatment are important stressors for HCWs. ${ }^{8}$ This may result into feelings of loneliness and helplessness, physical and mental fatigue, and depression or anxiety symptoms. Thus COVID-19 is a major challenge to mental health. The MHPs need to develop well-planned strategies at delivering mental health services to the affected population as well as strengthening stress coping abilities of the community to deal with the stress of social isolation and economic crisis in the background of the lockdown. There is also a strong need to tackle the stigma and discrimination being faced by the persons infected by COVID-19 and their contacts even following recovery. The MHPs can also play an important role in managing stigma in the community.

\section{Strategies for Developing Mental Health Care Services during Coronavirus Disease 2019 Pandemic}

COVID-19 pandemic is fast turning into a global mental health crisis and major international agencies have launched wide-ranging efforts to raise awareness about its mental health implications. WHO has been instrumental in highlighting specific mental health issues and assisting countries in formulating their response. ${ }^{9}$ On May 13, 2020, the United Nations (UN) Secretary General emphasized mental health as essential component of COVID-19 response and launched its first policy brief on "COVID-19 and the need for action on mental health." ${ }^{10} \mathrm{UN}$ has urged the governments, the civil society, and the health authorities to collaborate and urgently address the mental health dimension of the pandemic. The policy document recommends three immediate actions for this purpose: (1) a whole-of-society approach for mental health promotion and protection, (2) widespread availability of emergency mental health and psychosocial support, and (3) building mental health services for the future to support recovery from the pandemic. Academic institutions and health and social-sector organizations across the world have become active in conducting research and disseminating useful information focusing on strategies to cushion the enormous psychological impact of the COVID-19. Various national governments including the Government of India have also been taking steps for integrating mental health and psychosocial support as part of management plan for the pandemic. There is no single prescribed policy for this unprecedented event in modern history, and the strategies would evolve gradually with the progress of the global pandemic. MHPs and policy makers need to join forces to develop a comprehensive and multidimensional response plan. Building capacity for promotive, preventive and curative aspects of mental health care for society at large with focus on vulnerable groups is need of the hour. The strategies would include raising community awareness about the problem, expanding mental health services, emotional support for the quarantined persons, and supporting and sensitizing the health professionals.

\section{Raising Community Awareness}

Effective public health communication is a crucial step in controlling the escalation of overwhelming anxiety and panic. Basic knowledge about the modes of disease transmission and preventive measure should be made available to the public. Mass media including print, audiovisual, and social media are useful in dissemination of such information and educating the population. MHPs can be involved in developing and delivering such public health 
messages. ${ }^{11}$ Factually, accurate information in a simple, concise and comprehensible language coming from reliable sources (political leadership, health professionals, popular personalities, etc.) plays an important role in conveying the message. ${ }^{1}$ Equally important is countering the spread of misinformation and rumors by rapidly identifying malicious content, debunking myths, disseminating alerts, and counterarguments. Fear and anxiety due to COVID-19 infection can be addressed by unambiguous public messages from health authorities clearly describing prevention approaches, pathognomonic symptoms of COVID-19 infection, self-monitoring, and methods to swiftly access testing and medical help. In addition to the information about physical risks, public should also be educated about common psychological responses in such a situation and informed about methods of coping with stay at home restrictions (lockdown). ${ }^{12}$ Sharing experiences of individuals, who have recovered from COVID-19 infection, can also help to alleviate anxiety in community. Positive mental health and stress reduction strategies are useful in building resilience among individuals to tide over period of crisis. Basic advice includes sleep hygiene, activity scheduling, exercising, maintaining social connections, relaxation techniques, avoiding substance use and limiting time watching news and on social media. ${ }^{12}$

Social adversities like financial strain, loss of employment and educational opportunities, social isolation, and related issues secondary to the pandemic that threaten to trigger psychological distress also need to be addressed by raising awareness about the available alternatives for help, which may include welfare measures from the government and pragmatic financial advice. Options of work from home, distance learning using media, virtual classes from the educational institutions, and also virtually connecting with family members and friends are some other alternatives which can help in reducing distress and anxieties in the lockdown atmosphere. Another subject for community awareness programs is reduction of stigma associated with the infection and against those at higher risk of getting infected. ${ }^{7,9,13}$ Stigma reduction requires spreading the true facts, engagement of social influencers, amplifying the voices of people with lived experience of COVID-19 infection, honoring frontline care providers and promoting ethical journalism. ${ }^{14}$ Along with health information targeting the general population, specific advice and information support should be made available for the particularly vulnerable groups including children, elderly, people with COVID-19 infection, quarantined individuals, HCWs, people with preexisting mental disorders and those facing severe psychosocial adversities (e.g., migrants, unemployed and those facing domestic violence). Both national and international agencies like the WHO, ministry of health and family welfare, health care institutions, and professional societies are regularly publishing relevant public information about how to tackle various psychosocial issues arising out of the pandemic. Such information is available both in print as well as digital media in various local languages and needs to be further disseminated so as to reach all strata of the population.

\section{Expanding Mental Health Services}

COVID-19 pandemic has brought the issue of public mental health to the center of debate and offered a unique challenge for policymakers, academicians and clinicians globally. A major spike in psychological adversities and mental disorders is expected among general population and more so in the high-risk groups. In addition to lack of adequate human resource and infrastructure, risk of spread of infection has significantly disrupted the routine inpatient, outpatient, and community mental health services. Mental health systems across the world are quickly adapting to the situation by initiating and scaling up digital models of psychiatric assessments and consultations. ${ }^{15}$ Various audio-visual communication platforms and models are being used globally and evaluated for their effectiveness during the pandemic. On March 25, 2020, the board of governors of the Medical Council of India announced "Telemedicine Practice Guidelines" providing a legal framework enabling medical practitioners to practice telemedicine in the country. ${ }^{16}$ Subsequently, the All India Institute of Medical Sciences (AIIMS), New Delhi, started its telemedicine service for the follow-up patients. As a part of this service, the Department of Psychiatry, AIIMS, New Delhi, has also started telemedicine services. Given the uncertainty regarding the duration of pandemic, further innovative and sustainable models of telepsychiatry and telepsychotherapy services need to be developed and evaluated. Mobile application technology has also been used in past for many psychiatric disorders and has served as tools for health information, self-help, symptom monitoring, lifestyle modification, delivering psychotherapy and medication management. ${ }^{15}$ Many such apps are available on Internet and their use has been advocated during the pandemic. MHPs can recommend appropriate apps to patients which can become an integral part of treatment in future. Adopting digital psychiatry and scaling-up will require investment of resources, collaboration with IT professionals, training of clinicians and quality improvement based on experience..$^{15}$ Legal, ethical, and patient safety issues also need to be addressed while implementing such models.

It needs to be pointed out here that telemedicine can benefit mainly in routine consultations, that is, managing patients with low-risk and mild-to-moderate symptoms. Patients with severe symptoms or with comorbid physical health issues will require in person outpatient visits and may even need inpatient admission. It is therefore advisable that strict screening of patients, physical distancing, and infection control protocols are established for emergency, inpatient, and outpatient psychiatric settings. Further precautions would be needed in procedures like repetitive transmagnetic stimulation, electroconvulsive therapy which will pose higher risk to the treatment team and require extra precautions and personal protective equipments (PPEs). MHPs will need to be educated and trained in appropriate use of PPEs and self-monitoring of symptoms. Psychiatrists will also need to understand potential interactions of psychotropic medication with COVID-19 illness and its treatment, and prepare guidelines for the treatment of mental health problems in COVID positive patients. Since hospital inpatient units often become 
hotbeds of infection, it will be advisable to avoid inpatient admissions as much as possible and keep these reserved only for patients with life-threatening risk. The limitations placed on the hospital-based psychiatry system lay emphasis on development of community mental health. Reduction in inpatient admissions will require enhanced support for such patients in the community by creation of crisis intervention and home treatment teams as an alternative. Mental health workforce will need to be rapidly expanded to meet the rising demand, which can be done by increasing investment in community mental health. The National and District Mental Health Programs would need to be further strengthened to meet the challenge. As recommended by the UN and the WHO, mental health services would need to be integrated with COVID-19 response teams to improve access to a whole array of psychological and psychiatric services. ${ }^{10}$

\section{Support for the Quarantined Patients}

MHPs have another important role in COVID-19 units and quarantine centers. COVID-19 positive and suspected patients display a range of stress reactions which may interfere with their medical management. ${ }^{7}$ MHPs need to sensitize COVID-19 frontline HCWs about these issues and train them in communication skills to allay patient's anxiety, using screening tools for mental health problems, providing basic psychosocial support/counseling to patients and referring to a psychiatrist when needed. ${ }^{17}$ Health authorities should be proactive in their approach to address the mental health needs of patients and suspects to avoid untoward incidents. Quarantine centers and COVID-19 wards need to be equipped with patient education material about COVID-19 and mental health literature about managing stress and uncertainty. Structured daily routine, basic amenities, physical exercise, recreation facilities and encouraging virtual connectedness with family helps people cope with the quarantine. ${ }^{7,17}$ Various information technology-based models of providing mental health support to this population are currently being tested across the globe. Initial reports are encouraging and further research is needed to consolidate the findings and build sustainable models.

\section{Supporting and Sensitizing Health Professionals}

A large proportion of country's health care work force including doctors, nurses and paramedical staff are involved in provision of COVID-19 related services. They are experiencing significant psychological distress due to multiple factors and need to be sensitized and supported about need of caring for their own mental health. Studies are being done at national and international level to identify HCWs' needs, and to evaluate various psychological interventions to support them during this period. Health system leaders and hospital administrators need to understand the needs of the HCWs and create a positive work environment. ${ }^{18}$ These systemic interventions include adequate provision of PPEs, training in infection control, supervision and clinical protocols, adequate rest and sleep time, freedom of expression, motivation and boosting morale, opportunities to contact family members and availability of psychological help. ${ }^{17,18}$ MHPs have a key role in coordinating with COVID-19 response teams to screen HCWs for mental health problems, addressing their specific concerns, providing psychological support, teaching coping and self-care skills, problem-solving, ventilation and reassurance depending on the context of consultation. ${ }^{17}$ Dedicated confidential mental health helplines for health professionals providing access to both clinical psychologists and psychiatrists need to be made available.

\section{Conclusion}

COVID-19 pandemic is spreading alarmingly and has affected health, well-being and financial stability of the general population all over the world with significant impact on mental health. There is a need to raise awareness about its mental health implications and make strategies to manage the mental health issues. Effective public health communication in simple, concise, and comprehensible language coming from reliable sources may help to alleviate panic and anxiety in public. Mental health issues in persons being quarantined and in COVID-19 patients need to be taken care of. HCWs also face lot of stress while caring for the COVID-19 patient and need emotional support. IT-based technologies can be of great help in delivery of the mental health care. There is a need to strengthen the mental health system in view of various challenges and demand posed by COVID-19 pandemic

\section{Conflict of Interest}

None declared.

\section{References}

1 Coronavirus disease outbreak (COVID-19) outbreak situation report. Available at: https://www.who.int/emergencies/diseases/novel-coronavirus-2019?gclid=EAIaIQo bChMIwMLNs46w6QIVTx0rCh27RggHEAAYASAAEgKdn_D_ BwE. Accessed May 14, 2020

2 Covid-19 dashboard. Available at: https://www.mygov.in/ covid-19. Accessed May 14, 2020

3 Mak IW, Chu CM, Pan PC, Yiu MG, Chan VL. Long-term psychiatric morbidities among SARS survivors. Gen Hosp Psychiatry 2009;31(4):318-326

4 Xiang YT, Yang Y, Li W, et al. Timely mental health care for the 2019 novel coronavirus outbreak is urgently needed. Lancet Psychiatry 2020;7(3):228-229

5 Wang C, Pan R, Wan X, et al. Immediate psychological responses and associated factors during the initial stage of the 2019 Coronavirus disease (COVID-19) epidemic among the general population in China. Int J Environ Res Public Health 2020;17(5):1729

6 Liang L, Ren H, Cao R, et al. The effect of Covid-19 on youth mental health. Psychiatr Q 2020;1-2

7 Brooks SK, Webster RK, Smith LE, et al. The psychological impact of quarantine and how to reduce it: rapid review of the evidence. Lancet 2020;395(10227):912-920

8 Lai J, Ma S, Wang Y, et al. Factors associated with mental health outcomes among health care workers exposed to coronavirus disease 2019. JAMA Netw Open 2020;3(3):e203976

9 World Health Organization. (2020). Mental health and psychosocial considerations during the COVID-19 outbreak, 18 March 2020. Available at: https://www.who.int/publications-detail/ WHO-2019-nCoV-MentalHealth-2020.1 Accessed May 15, 2020 
10 United Nations. (2020). Policy Brief: COVID-19 and the Need for Action on Mental Health, 13 May 2020. Available at: https://www.un.org/sites/un2.un.org/ files/un_policy_briefcovid_and_mental_health_final.pdf. Accessed May 15, 2020

11 Bavel JJ, Baicker K, Boggio PS, et al. Using social and behavioural science to support COVID-19 pandemic response. Nat Hum Behav 2020;4(5):460-471

12 Banerjee D. The COVID-19 outbreak: crucial role the psychiatrists can play. Asian J Psychiatr 2020;50:102014

13 Ho CS, Chee CY, Ho RC. Mental health strategies to combat the psychological impact of covid-19 beyond paranoia and panic. Ann Acad Med Singapore 2020;49(3):155-160

14 UNICEF. (2020). Social stigma associated with the coronavirus disease (COVID-19): A guide to preventing and addressing social stigma, March 2020. Available at: https://www.unicef. org/documents/social-stigma-associated-coronavirus-disease-co vid-19. Accessed May 15, 2020
15 Torous J, Jän Myrick K, Rauseo-Ricupero N, Firth J. Digital mental health and Covid-19: using technology today to accelerate the curve on access and quality tomorrow. JMIR Ment Health 2020;7(3):e18848

16 Ministry of Health and Family Welfare. (2020). Telemedicine practice guidelines enabling registered medical practitioners to provide healthcare using telemedicine. Available at: https:// www.mohfw.gov.in/pdf/Telemedicine.pdf. Accessed 15, May 2020

17 Grover S, Dua D, Sahoo S, Mehra A, Nehra R, Chakrabarti S. Why all COVID-19 hospitals should have mental health professionals: The importance of mental health in a worldwide crisis! Asian J Psychiatr 2020;51:102147

18 Greenberg N, Docherty M, Gnanapragasam S, Wessely S. Managing mental health challenges faced by healthcare workers during covid-19 pandemic. BMJ 2020;368:m1211 